\title{
Acute and one-year clinical outcomes following implantation of bioresorbable vascular scaffolds: the ABSORB UK Registry
}

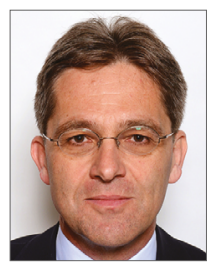

Andreas Baumbach ${ }^{1,2 *}$, MD; Azfar Zaman³, MD; Nick E.J. West', MD; Peter O'Kane ${ }^{5}$, MD; Mohaned Egred $^{3}, \mathrm{MD}$; Thomas Johnson ${ }^{2}, \mathrm{MD}$; Stephen Wheatcroft ${ }^{6}, \mathrm{MD}$; Ruth Bowles ${ }^{2}, \mathrm{BSc}$; Adam de Belder ${ }^{7}, \mathrm{MD}$; Georgios Bouras ${ }^{8}$, MD; Alexandra Lansky ${ }^{1,8}$, MD; Jonathan Hill ${ }^{9}, \mathrm{MD}$; Anthony Mathur ${ }^{1}$, MD; Mark A. de Belder ${ }^{10}, \mathrm{MD}$; Adrian P. Banning ${ }^{11}$, MD; for the ABSORB UK investigators

1. Barts Heart Centre, William Harvey Research Institute, Queen Mary University of London, London, United Kingdom; 2. Bristol Heart Institute, University Hospitals Bristol, Bristol, United Kingdom; 3. Department of Cardiology, Freeman Hospital, Newcastle University, Newcastle-upon-Tyne, United Kingdom; 4. Department of Interventional Cardiology, Papworth Hospital, Cambridge, United Kingdom; 5. Dorset Heart Centre, Royal Bournemouth Hospital, Bournemouth, United Kingdom; 6. Leeds Multidisciplinary Cardiovascular Research Centre, University of Leeds, Leeds, United Kingdom; 7. Sussex Cardiac Centre, Brighton and Sussex University Hospitals NHS Trust, Brighton, United Kingdom; 8. Section of Cardiology, Department of Medicine, Yale University School of Medicine, New Haven, CT, USA; 9. Department of Cardiology, King's College Hospital, London, United Kingdom; 10. The James Cook University Hospital, Middlesbrough, United Kingdom; 11. Oxford Heart Centre, John Radcliffe Hospital, Oxford, United Kingdom

The accompanying supplementary data are published online at: http://www.pcronline.com/eurointervention/128th_issue/250

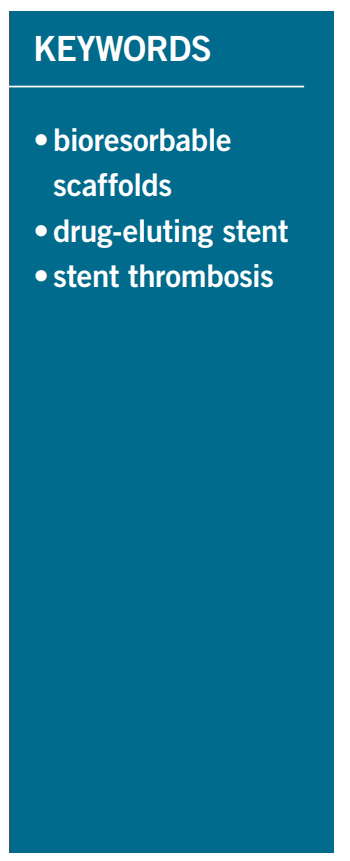

\begin{abstract}
Aims: The aim of this registry was to monitor practice patterns and outcomes in patients treated with Absorb bioresorbable vascular scaffolds (BVS) during the early phase of usage in the UK.

Methods and results: A total of 1,005 patients with de novo coronary lesions were treated using careful implantation techniques at 24 centres. Follow-up at one year was obtained in $99 \%$. An independent clinical events committee and angiographic core lab adjudicated all events. Patient age was 52 years and $46 \%$ presented with acute coronary syndromes. Lesion complexity was B2/C in $47.4 \%$, with an average of $1.4 \pm 0.6$ BVS implanted and a BVS length of $28 \pm 14.3 \mathrm{~mm}$. A single BVS was implanted in $71.3 \%$. High pressure post-dilatation was performed in $94.9 \%$, with $50 \%$ using intravascular imaging. Device success was achieved in $98.7 \%$ and procedure success in $97.3 \%$ of patients. At one year, the device-oriented endpoint target lesion failure was 3.2\%, and the patient-oriented endpoint major adverse cardiac events was $3.4 \%$. Definite ST occurred in 1.4\%. By multivariable analysis, scaffold size of $2.5 \mathrm{~mm}$ predicted ST at one year (OR 3.27, 95\% CI: 1.28-8.37; $\mathrm{p}=0.014$ ).
\end{abstract}

Conclusions: With careful patient selection and technique, TLF rates following BVS implantation in realworld practice are comparable with contemporary drug-eluting stents. ST rates remain high, with small vessel size identified as a contributory factor. ClinicalTrials.gov Identifier: NCT01977534

\footnotetext{
*Corresponding author: Barts Heart Centre, West Smithfield, London, EC1A 7BE, United Kingdom. E-mail:a.baumbach@qmul.ac.uk
} 


$\begin{array}{ll}\text { Abbreviations } \\ \text { BVS } & \text { bioresorbable vascular scaffold } \\ \text { DAPT } & \text { dual antiplatelet therapy } \\ \text { DES } & \text { drug-eluting stent } \\ \text { IVUS } & \text { intravascular ultrasound } \\ \text { MACE } & \text { major adverse cardiac events } \\ \text { OCT } & \text { optical coherence tomography } \\ \text { QCA } & \text { quantitative coronary analysis } \\ \text { ST } & \text { stent thrombosis } \\ \text { TLF } & \text { target lesion failure } \\ \text { TVF } & \text { target vessel failure }\end{array}$

\section{Introduction}

The transition from controlled initial feasibility studies and early randomised trials with restricted inclusion criteria to use in everyday clinical practice requires the careful assessment of clinical outcomes achieved by new devices. Fully bioresorbable scaffolds were introduced as an alternative to metallic coronary stents with the aim of limiting the perceived shortcomings of permanent metallic implants. The device with the largest clinical evidence is the Absorb $^{\mathrm{TM}}$ everolimus-eluting bioresorbable scaffold (BVS; Abbott Vascular, Santa Clara, CA, USA) - a balloon-expandable bioresorbable scaffold consisting of a poly-L-lactide backbone coated with a 1:1 mixture of polyD,L-lactide and everolimus.

Whilst long-term outcomes from the restricted early feasibility cohorts have suggested efficacy and safety up to five years ${ }^{1}$, later experience in randomised controlled trials was ambiguous, demonstrating non-inferiority in efficiency but a safety concern with increased rates of early and late stent thrombosis (ST) compared with implantation of metallic everolimus-eluting drug-eluting stents (DES $)^{2-5}$.

The BVS gained European marketing approval (CE mark) in 2011 and was approved by the US FDA in $2016^{6}$, now with over 150,000 BVS implants worldwide. Following market introduction, it is essential to monitor clinical practice and long-term outcomes in broader populations representative of routine clinical practice ${ }^{7}$. Several post-market registries have been designed for this purpose in various countries and as multinational initiatives ${ }^{8-10}$. Reports from early experience in realworld clinical practice raised concern over increased rates of adverse events related to patient selection and implantation technique ${ }^{11}$. The Absorb UK Registry was initiated in 2013, at a time when early concerns and experience were already acknowledged, but before the potential for implant technique to mitigate early events had been described ${ }^{12}$. The aim of the ABSORB UK Registry was to monitor practice patterns and clinical outcomes in the early phase of technology rollout in this country, with an emphasis on careful patient and lesion selection, good implantation technique and high data quality with complete follow-up to provide a meaningful assessment of real-world outcomes with contemporary use of BVS.

Editorial, see page 1506

\section{Methods}

The ABSORB UK Registry is an investigator-initiated, prospective multicentre post-market registry evaluating the Absorb BVS in patients with symptomatic de novo native coronary artery lesions.

\section{PATIENT POPULATION}

Patients with a clinical indication for coronary revascularisation, a de novo lesion and treatment with at least one Absorb BVS were eligible. General inclusion criteria were age of at least 18 years at the time of signing the informed consent form, and treatment of de novo lesions located in previously untreated vessels; prior treatment of a lesion in a non-target vessel was permitted. Patients had to agree to undergo all required follow-up visits and data collection. General exclusion criteria were inability to give informed consent or comply with the protocol as per the investigator's judgement. Patients meeting all inclusion and no exclusion criteria were asked to participate in the registry. The trial was performed in accordance with the Declaration of Helsinki, with ethical approval, and all patients signed consent forms to participate in the registry.

\section{PROCEDURE AND IMPLANTATION TECHNIQUE}

The steering group together with an advisory board issued recommendations to all participating centres and implanting operators at the initiation of the registry, based on the best available evidence at the time for BVS, for optimal procedural technique and patient selection. The selection criteria were classified as "favourable" (young patients $<65$ yrs at risk for future interventions, simple straightforward lesions, proximal/mid LAD, multivessel disease, diabetes), "with caution" (STEMI, bifurcations, long diffuse disease, saphenous vein grafts, ostial lesions, calcification, tortuosity, in-stent stenosis, chronic total occlusion), and "best avoided" (vessel sizes $<2.5$ and $>4.0 \mathrm{~mm}$, true bifurcations requiring two stents, heavy calcification, dual antiplatelet therapy [DAPT] $<12$ months, left main stem).

The technical recommendation was for appropriate lesion preparation with low residual stenosis prior to implantation of the scaffold, sizing according to angiographic vessel size with avoidance of undersizing, and routine post-dilatation with high-pressure noncompliant balloons. The use of online quantitative coronary analysis (QCA) was explicitly encouraged in order to size the scaffold appropriately. The vessel size range was governed by the available scaffold sizes and the limited capacity for further expansion after implantation. Adjunctive imaging with IVUS or OCT was not specifically recommended but encouraged for complex lesions.

Selection, dosing and duration of antiplatetet therapy was left to individual operators' discretion. The majority of centres opted for a prescribed DAPT duration of 12 months.

\section{FOLLOW-UP AND OUTCOME DEFINITIONS}

Outcomes were assessed in hospital and at 12 months by office or telephone contact. For patients who could not be reached by telephone, the patient's primary care physician was contacted. Extended follow-up is planned to three years. 
The primary endpoint of the study was the device-oriented outcome of target lesion failure (TLF) at 12 months, defined as the composite of cardiac death, target vessel MI and ischaemia-driven target lesion revascularisation (TLR). Additional device-related outcomes included target vessel failure (TVF) and target vessel revascularisation (TVR). TVF included cardiac death, all MI and ischaemia-driven TVR. The main patient-related outcome was major adverse cardiac events (MACE), defined as cardiac death, all MI, and ischaemia-driven TLR. Staged planned procedures during the follow-up period were identified and not included in MACE reporting unless they involved the target lesion.

Components of the composite outcomes were reported at each time point and included death (cardiovascular and non-cardiovascular), MI (target and non-target vessel-related and based on a protocol definition of symptoms and development of a new Q-wave on ECG or a rise of biomarkers of $>2$ times the upper limit of normal and separately according to the third universal definition of myocardial infarction), TLR (all and ischaemia-driven), TVR (all and ischaemia-driven), all coronary revascularisations and ST based on Academic Research Consortium (ARC) definitions ${ }^{13}$.

Procedural outcomes included acute success, defined as successful implantation of one or more scaffolds with a final inscaffold residual diameter stenosis of $<50 \%$, without BVS device deficiency. Clinical procedural success was defined as successful implantation of one or more BVS with achievement of a final inscaffold diameter stenosis of $<50 \%$, without target vessel failure within three days of the index procedure. "PSP" was considered according to the available data when predilatation was performed, sizing was appropriate by operator assessment, and post-dilatation with high pressure $\geq 16$ atm was completed.

An independent clinical events committee, consisting of experienced and unbiased cardiologists, adjudicated all serious adverse events and protocol endpoints. While QCA was not available for all procedures, all repeat coronary procedures, including repeat angiograms, or planned staged coronary interventions were adjudicated and analysed by an independent angiographic core laboratory (Yale Cardiovascular Research Group, New Haven, CT, USA).

The steering committee reviewed and provided guidance on the protocol, site and patient selection, procedural technique, data collection and publications. The study sponsor was the Bristol Heart Institute, and data management was supported by Abbott Vascular. The registry was sponsored by Abbott Vascular with an unrestricted grant.

\section{STATISTICAL ANALYSES}

The ABSORB UK sample size was not defined on the basis of an endpoint hypothesis but rather to monitor practice patterns and clinical outcomes in the early phase of BVS usage in the UK and should be seen as hypothesis-generating. All analyses were performed on all successfully registered patients.

Continuous variables are summarised using mean \pm standard deviation (SD) or median and interquartile ranges (IQR) as appropriate, and 95\% confidence intervals were calculated using Gaussian approximation. Categorical parameters are reported as counts and percentages and exact $95 \%$ confidence intervals calculated using the Clopper-Pearson method.

To determine the independent predictors of TLF, MACE and ST, a univariate analysis was performed and then a multivariable logistic regression model was built using a stepwise (forward/backward) procedure, with independent variables entered into the model at the 0.20 significance level and removed at the 0.10 level. Variables were eligible for inclusion in the multivariable logistic regression model-building process if the variable was present for $90 \%$ of the subjects in the analyses, and if they had a p-value $<0.2$ from the univariable analysis; if highly correlated with another variable $(r>0.5$ and $\mathrm{p}<0.05$ ), the variable with higher level of significance was included. With this approach the number of covariates eligible was increased. The univariable logistic regression reported in the Supplementary data depicts the number of covariates initially considered.

Time-to-event TLF is presented as a Kaplan-Meier curve.

A two-sided p-value of less than 0.05 indicated statistical significance.

\section{Results}

Between January 2014 and October 2015, 1,005 patients were enrolled at 24 centres. The number of patients per centre ranged from one to 150 (Supplementary Table 1). BVS implantation accounted for $2.7 \%$ (range $0.2-7.3 \%$ ) of total PCI in centres.

Patient baseline, angiographic characteristics and procedural data are listed in Table 1 and Table 2. The mean age was 52 years, and $13.8 \%$ presented with STEMI. Lesion complexity was classified as $\mathrm{B} 2 / \mathrm{C}$ in $47.4 \%$ by operator assessment.

\section{PROCEDURE AND TECHNIQUE}

Lesion characteristics and procedural details are listed in Table 3. The average number of lesions treated was $1.3 \pm 0.6$ with a mean of 1.4 \pm 0.6 BVS implants per patient and a total BVS length of $28 \pm 14.3 \mathrm{~mm}$. The proportion of patients receiving one BVS was

\section{Table 1. Patient baseline demographics and clinical} characteristics.

\begin{tabular}{|l|c|}
\hline Number & 1,005 \\
\hline Age & $52 \pm 11 \mathrm{yrs}$ \\
\hline Male & $754(75 \%)$ \\
\hline Smoker & $290(28.9 \%)$ \\
\hline Hypertension & $501(49.9 \%)$ \\
\hline Dyslipidaemia & $568(56.5 \%)$ \\
\hline Diabetes & $175(17.4 \%)$ \\
\hline Prior MI & $244(24.3 \%)$ \\
\hline Prior PCl & $210(20.9 \%)$ \\
\hline Prior CABG & $13(1.3 \%)$ \\
\hline NSTEMI & $321(31.9 \%)$ \\
\hline STEMI & $139(13.8 \%)$ \\
\hline $\begin{array}{l}\text { CABG: coronary artery bypass graft; MI: myocardial infarction; } \\
\text { NSTEMI: non-ST-elevation myocardial infarction; PCI: percutaneous } \\
\text { coronary intervention; STEMI: ST-elevation myocardial infarction }\end{array}$ \\
\hline
\end{tabular}


Table 2. Angiographic and procedural characteristics.

\begin{tabular}{|c|c|c|}
\hline \multicolumn{2}{|l|}{ Number of patients } & 1,005 \\
\hline \multicolumn{2}{|l|}{ Number of lesions } & 1,263 \\
\hline \multicolumn{2}{|l|}{ Single-vessel disease } & $65.8 \%$ \\
\hline \multicolumn{2}{|l|}{ Multivessel disease } & $19 \%$ \\
\hline \multicolumn{2}{|l|}{ LAD } & $54 \%$ \\
\hline \multicolumn{2}{|l|}{$\mathrm{RCA}$} & $27 \%$ \\
\hline \multicolumn{2}{|l|}{ LCx } & $18 \%$ \\
\hline \multicolumn{2}{|l|}{ Left main stem } & $0.7 \%$ \\
\hline \multicolumn{2}{|l|}{ Reference diameter } & $3.16 \pm 0.46 \mathrm{~mm}$ \\
\hline \multicolumn{2}{|l|}{ Number of lesions treated } & $1.3 \pm 06$ \\
\hline \multirow[t]{3}{*}{ Number of BVS } & 1 & $71.3 \%$ \\
\hline & 2 & $22.4 \%$ \\
\hline & $3+$ & $6.2 \%$ \\
\hline \multicolumn{2}{|l|}{ Radial access } & $89.3 \%$ \\
\hline \multirow[t]{4}{*}{ Antiplatelet agent } & Aspirin & $99.9 \%$ \\
\hline & Clopidogrel & $59.8 \%$ \\
\hline & Ticagrelor & $32.6 \%$ \\
\hline & Prasugrel & $7.6 \%$ \\
\hline \multicolumn{2}{|l|}{ Predilatation } & $97.5 \%$ \\
\hline \multicolumn{2}{|l|}{ Predilatation balloon diameter } & $2.9 \pm 1.41 \mathrm{~mm}$ \\
\hline \multirow[t]{3}{*}{ Lesion preparation } & Rotablation & $0.7 \%$ \\
\hline & Cutting balloon & $11.8 \%$ \\
\hline & Other & $0.7 \%$ \\
\hline \multicolumn{2}{|l|}{ Post-dilatation with NC balloon } & $94.9 \%$ \\
\hline \multicolumn{2}{|l|}{ Post-dilatation balloon diameter } & $3.46 \pm 1.01 \mathrm{~mm}$ \\
\hline \multicolumn{2}{|l|}{ Post-dilatation pressure } & $17.0 \pm 3.8 \mathrm{~atm}$ \\
\hline \multicolumn{2}{|l|}{ PSP } & $65.8 \%$ \\
\hline \multirow[t]{3}{*}{ Use of intracoronary imaging } & Overall & $50 \%$ \\
\hline & IVUS & $13.3 \%$ \\
\hline & OCT & $40.1 \%$ \\
\hline \multicolumn{2}{|l|}{ Clinical procedure success } & $97.3 \%$ \\
\hline \multicolumn{2}{|l|}{ Clinical device success } & $98.7 \%$ \\
\hline \multicolumn{3}{|c|}{$\begin{array}{l}\text { BVS: bioresorbable scaffold; IVUS: intravascular ultrasound; LAD: left } \\
\text { anterior descending artery; LCx: left circumflex artery; NC: non- } \\
\text { compliant; OCT: optical coherence tomography; PSP: predilatation, } \\
\text { sizing according to reference diameter, post-dilatation with high- } \\
\text { pressure NC balloon > } 16 \text { atm; RCA: right coronary artery }\end{array}$} \\
\hline
\end{tabular}

$71.3 \%$, and $22.4 \%$ received two scaffolds. The mean scaffold diameter was $3.1 \pm 0.37 \mathrm{~mm}$.

Predilatation (mean balloon diameter $3.0 \mathrm{~mm}$ ) was performed in $97.5 \%$ of lesions; cases without predilatation were limited to patients presenting with STEMI or spontaneous coronary artery dissection. Post-dilatation with high-pressure non-compliant balloons was performed in $94.9 \%$ at a mean pressure of 17 atmospheres. Intravascular imaging, mostly OCT, was used in $50 \%$ of cases.

\section{OUTCOMES}

Complete follow-up at one year was available for 992 patients (98.7\%). Clinical endpoints are tabulated for in-hospital, 30-day and one-year time points in Table 4. Acute device success was achieved in $98.7 \%$ of lesions, and clinical procedural success was achieved in $97.3 \%$ of patients. At one year, the TLF rate was $3.2 \%$ (Figure 1), the rate of MACE was $3.4 \%$, and the definite ST rate was $1.4 \%$ (acute $0.1 \%$, subacute $0.7 \%$, late $0.6 \%$ ). An independent
Table 3. Lesion characteristics and scaffold implantation.

\begin{tabular}{|c|c|c|}
\hline \multirow[t]{4}{*}{ Lesion type } & A & $17.8 \%$ \\
\hline & B1 & $34.8 \%$ \\
\hline & B2 & $21.5 \%$ \\
\hline & C & $25.9 \%$ \\
\hline \multicolumn{2}{|l|}{ De novo lesion } & $99.3 \%$ \\
\hline \multicolumn{2}{|l|}{ Restenosis } & $0.4 \%$ \\
\hline \multicolumn{2}{|l|}{ Lesion length } & $23.3 \pm 13.3 \mathrm{~mm}$ \\
\hline \multicolumn{2}{|l|}{ Bifurcation } & $15.1 \%$ \\
\hline \multicolumn{2}{|l|}{ Total occlusion } & $7.7 \%$ \\
\hline \multicolumn{2}{|l|}{ Ostial lesion } & $7.2 \%$ \\
\hline \multicolumn{2}{|l|}{ Vessel tortuosity } & $8.9 \%$ \\
\hline \multicolumn{2}{|c|}{ Calcification (moderate/severe) } & $8.2 \%$ \\
\hline \multicolumn{2}{|c|}{ Number of BVS used per lesion } & $1.4 \pm 06$ \\
\hline \multicolumn{2}{|c|}{ Total length of BVS [range] } & $28 \pm 14.3 \mathrm{~mm}[12,102 \mathrm{~mm}]$ \\
\hline \multirow[t]{3}{*}{ BVS diameter } & $2.5 \mathrm{~mm}$ & $22.3 \%$ \\
\hline & $3.0 \mathrm{~mm}$ & $37.8 \%$ \\
\hline & $3.5 \mathrm{~mm}$ & $39.9 \%$ \\
\hline \multirow[t]{3}{*}{ BVS length } & $12 \mathrm{~mm}$ & $15.4 \%$ \\
\hline & $18 \mathrm{~mm}$ & $38.3 \%$ \\
\hline & $28 \mathrm{~mm}$ & $46.3 \%$ \\
\hline \multicolumn{2}{|c|}{ Patients with overlapping BVS } & $33.2 \%$ \\
\hline
\end{tabular}

Table 4. Clinical outcomes in-hospital, at 30-day and at one-year follow-up.

\begin{tabular}{|c|c|c|c|c|}
\hline & $\begin{array}{c}\text { In hospital } \\
N=1,005\end{array}$ & $\begin{array}{l}30 \text { days } \\
N=992\end{array}$ & $\begin{array}{c}12 \text { months } \\
\mathrm{N}=992\end{array}$ \\
\hline \multicolumn{2}{|l|}{ Target lesion failure } & $9(0.9 \%)$ & $12(1.2 \%)$ & $32(3.2 \%)$ \\
\hline \multicolumn{2}{|l|}{ MACE } & $9(0.9 \%)$ & $12(1.2 \%)$ & $34(3.4 \%)$ \\
\hline \multicolumn{2}{|l|}{ Target vessel failure } & $10(1.0 \%)$ & $14(1.4 \%)$ & $43(4.3 \%)$ \\
\hline \multicolumn{2}{|l|}{ All death } & $0(0 \%)$ & $1(0.1 \%)$ & $6(0.6 \%)$ \\
\hline \multicolumn{2}{|l|}{ Cardiac death } & $0(0 \%)$ & $1(0.1 \%)$ & $3(0.3 \%)$ \\
\hline \multirow{3}{*}{$\begin{array}{l}\text { MI (protocol } \\
\text { definition) }\end{array}$} & All MI & $8(0.8 \%)$ & $10(1 \%)$ & $21(2.1 \%)$ \\
\hline & Q-wave MI & $5(0.5 \%)$ & $7(0.7 \%)$ & $8(0.8 \%)$ \\
\hline & Non-Q-wave MI & $3(0.3 \%)$ & $3(0.3 \%)$ & $13(1.3 \%)$ \\
\hline \multirow{6}{*}{$\begin{array}{l}\text { MI (third universal } \\
\text { definition) }\end{array}$} & Type 1 & $2(0.2 \%)$ & $4(0.4 \%)$ & $15(1.5 \%)$ \\
\hline & Type 2 & $2(0.2 \%)$ & $2(0.2 \%)$ & $4(0.4 \%)$ \\
\hline & Type 3 & $0(0 \%)$ & $0(0 \%)$ & $1(0.1 \%)$ \\
\hline & Type 4a & $15(1.5 \%)$ & $15(1.5 \%)$ & $17(1.7 \%)$ \\
\hline & Type 4b & $6(0.6 \%)$ & $8(0.8 \%)$ & $13(1.3 \%)$ \\
\hline & Type 5 & $0(0 \%)$ & $0(0 \%)$ & $0(0 \%)$ \\
\hline \multirow{4}{*}{$\begin{array}{l}\text { Target lesion } \\
\text { revascularisation }\end{array}$} & All & $6(0.6 \%)$ & $10(1.0 \%)$ & $25(2.5 \%)$ \\
\hline & ID-TLR & $6(0.6 \%)$ & $9(0.9 \%)$ & $23(2.3 \%)$ \\
\hline & CABG & $0(0 \%)$ & $0(0 \%)$ & $1(0.1 \%)$ \\
\hline & $\mathrm{PCl}$ & $6(0.6 \%)$ & $9(0.9 \%)$ & $22(2.2 \%)$ \\
\hline \multirow{4}{*}{$\begin{array}{l}\text { Target vessel } \\
\text { revascularisation }\end{array}$} & All & $8(0.8 \%)$ & $13(1.3 \%)$ & $38(3.8 \%)$ \\
\hline & ID-TVR & $8(0.8 \%)$ & $12(1.2 \%)$ & $36(3.6 \%)$ \\
\hline & CABG & $0(0 \%)$ & $0(0 \%)$ & $4(0.4 \%)$ \\
\hline & $\mathrm{PCl}$ & $8(0.8 \%)$ & $12(1.2 \%)$ & $32(3.2 \%)$ \\
\hline \multirow[t]{2}{*}{ All revascularisation } & $\mathrm{PCl}$ & $19(1.9 \%)$ & $38(3.8 \%)$ & $143(14.3 \%)$ \\
\hline & CABG & $0(0 \%)$ & $0(0 \%)$ & $6(0.6 \%)$ \\
\hline \multicolumn{2}{|c|}{ Stent thrombosis (definite/probable)* } & $\begin{array}{c}\text { Acute }(<1 \mathrm{~d}) \\
2(0.2 \%)\end{array}$ & $9(0.9 \%)$ & $17(1.7 \%)$ \\
\hline \multicolumn{5}{|c|}{$\begin{array}{l}\text { *Core lab adjudicated. CABG: coronary artery bypass grafting; ID-TLR: ischaemia-driven } \\
\text { target lesion revascularisation; ID-TVR: ischaemia-driven target vessel revascularisation; } \\
\text { MACE: major adverse cardiac events; MI: myocardial infarction; PCI: percutaneous coronary } \\
\text { intervention }\end{array}$} \\
\hline
\end{tabular}




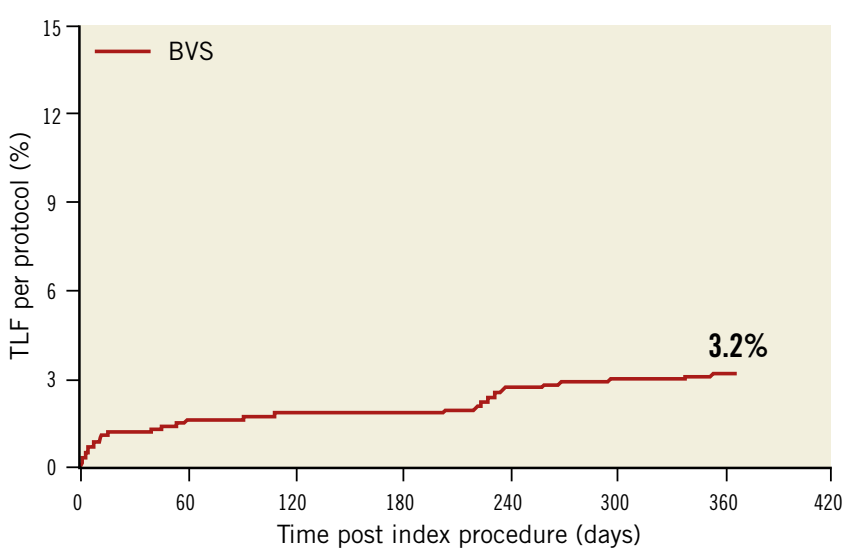

Figure 1. Kaplan-Meier curve representing the estimated cumulative incidence rate of TLF up to one year (365 days) - per subject analysis. TLF: target lesion failure

angiographic core laboratory confirmed all ST. The average vessel diameter of these 14 cases was $2.62 \pm 0.41 \mathrm{~mm}$, the scaffold size $2.62 \pm 0.45 \mathrm{~mm}$, and the length was $16.13 \pm 8.85 \mathrm{~mm}$. In $5 / 14$ cases with definite ST, the core lab identified the target vessel to be less than $2.3 \mathrm{~mm}$ in diameter (range: 1.8-2.25 $\mathrm{mm}$ ) by QCA, and in $2 / 14$ cases there was a marked size mismatch (scaffold undersized $>0.2 \mathrm{~mm}$ ).

By multivariable analysis the only independent predictor of TLF was increasing age (OR 0.96, 95\% CI: 0.93-1.0; $\mathrm{p}=0.0337$ ). There were no predictors of MACE.

Correlates of ST identified in univariate analysis included scaffold size (2.5 mm; OR 3.26, 95\% CI: 1.27-8.35; $\mathrm{p}=0.0137)$, moderate/severe calcification (OR 3.11, 95\% CI: 1.00-9.67; $\mathrm{p}=0.0497$ ) and BVS procedure volume by centre $(\leq 20$; OR $4.47,95 \%$ CI: $1.31-15.2 ; \mathrm{p}=0.0166$ ).

Only scaffold size of $2.5 \mathrm{~mm}$ predicted ST at one year (OR $3.27,95 \%$ CI: $1.28-8.37 ; \mathrm{p}=0.0136$ ) by multivariable analysis. Neither the use of imaging, nor achievement of PSP showed a correlation with reduced TLF, ST or MACE in the univariate analysis. Univariate analysis results are provided in detail for TLF, MACE and ST in Supplementary Table 2-Supplementary Table 4.

\section{Discussion}

The major findings of ABSORB UK were a very low rate of TLF (3.2\%) at one year comparable to current drug-eluting stent results, and a rate of definite ST of $1.4 \%$. Implantation technique included $50 \%$ use of intravascular imaging and high-pressure post-dilatation in $95 \%$ of cases. Angiographic core lab assessment revealed that at least half of stent thromboses (7/14) were related to small vessel size or undersizing of the scaffold. Importantly, this real-world UK registry represents a range of operator experience and procedural techniques of BVS implantation during the early rollout of this technology. The registry was performed with high-quality data ( $99 \%$ clinical follow-up), independent adjudication of clinical events and angiographic core lab review of all repeat angiography and interventions.

\section{IMPLANTATION TECHNIQUE}

The ABSORB UK Registry began enrolling in 2014, later than most other registries and at a time when experience with scaffold implantation was widely discussed. Therefore, special care was taken to advise the operators about the importance of lesion selection, predilatation to achieve complete lesion preparation, sizing to avoid mismatch and post-dilatation to achieve full scaffold expansion. The technique of "Predilatation, Sizing, Postdilatation" (PSP), however, had not been established at the time. Whilst in prior clinical trials high-pressure post-dilatation had been discouraged over concerns of scaffold fracture ${ }^{8,14}$, it was strongly encouraged at the beginning of our enrolment. Likewise, guidance by intracoronary imaging, while not mandatory, was encouraged particularly in cases with more complex lesion anatomy.

\section{STENT THROMBOSIS}

A discrepancy between results achieved in the early cohorts, randomised controlled trials (RCT) and real-world registries was first brought to attention in the GHOST-EU registry ${ }^{11}$. Higher rates of ST were linked to suboptimal implantation technique and treatment of complex lesions ${ }^{15,16}$. In the ABSORB III randomised trial which enrolled patients in centres with no prior experience with the technology, rates of thrombosis at one year were numerically higher in the BVS group (1.5\%) than in the DES group $(0.7 \%)^{5}$, linked principally to use in small vessels $(<2.25 \mathrm{~mm})$. A recent all-comers RCT performed in highly experienced centres in the Netherlands found a significantly increased ST rate at two years $(3.5 \%)$ in a cohort with increased lesion complexity ${ }^{17}$.

\section{FACTORS ASSOCIATED WITH LONG-TERM OUTCOME}

The ABSORB UK Registry mirrors this experience with an increased ST rate of $1.4 \%$. We found an association between centre contribution, vessel calcification and small scaffold size and an increased rate of ST. In multivariable analysis, only the use of the smallest scaffold size remained significantly correlated to ST. Based on the independent core lab analysis of repeat angiography with the corresponding index implantation angiograms, we were able to define further small target vessels $(<2.25 \mathrm{~mm})$ or scaffold undersizing as a contributor to subsequent ST. This observation suggests that even greater care has to go into selection of target vessels within the appropriate range and sizing using the best available technology.

During the period of this registry, increasing attention has been given to the PSP implantation technique ${ }^{18}$. We were not able to document a correlation between PSP and outcomes. However, this is most likely due to a lack of prospective use of this technique, absence of QCA analysis for all vessels and the fact that the definition of PSP has evolved. Very high post-dilatation pressure and the use of post-dilatation with balloons larger than the scaffold size has recently been advocated ${ }^{19}$.

OCT or IVUS was used in $50 \%$ of cases and $37.5 \%$ of operators reported additional treatment or an altered procedural strategy 
based on invasive imaging. Nevertheless, we were not able to demonstrate a benefit towards reduced adverse events in our cohort. There was no routine use of intracoronary imaging in cases of ST, and hence no further morphological information could be gained from these cases.

At one year, 984 patients (99.1\%) were still receiving DAPT. Hence, we cannot attribute the incidence of ST to DAPT cessation or interruption. No formal recommendation has been issued by the steering group with a view to the duration of DAPT and it remains to be seen whether extended use beyond 12 months will impact on the incidence of very late ST.

\section{OUTLOOK}

The very low rate of TLF in our cohort of patients points towards good medium-term performance of the implanted scaffolds. The benefit of BVS over DES was thought to manifest itself with an absence of long-term adverse events after absorption of the scaffold itself. For this reason, follow-up at three years is currently ongoing and will provide further information about the long-term safety and efficacy of the current BVS platform.

\section{Limitations}

The ABSORB UK Registry is a prospective registry, not a randomised trial. The recommendations for patient selection and implantation technique were not binding. A prolonged approval process in some centres led to small enrolment numbers in these sites and hence inclusion of an early individual learning curve. Although consecutive enrolment of BVS implants was requested, this was not monitored. For all these reasons, some selection bias towards reporting in the registry cannot be ruled out.

\section{Conclusions}

Implantation of fully bioresorbable vascular scaffolds requires good lesion selection and careful implantation technique to provide good long-term outcomes. This is the first large-scale real-world registry in which implantation technique followed contemporary recommendations with the use of imaging and high-pressure post-dilatation. One-year data show a high efficiency. An excess of ST was linked to cases with inadequate sizing. Three-year follow-up will provide important information on the longevity of the achieved outcomes and absence of further cases of ST.

\section{Impact on daily practice}

In the light of the recent withdrawl of this particular scaffold, the results will inform practice and design of trials for futuregeneration devices.

\section{Acknowledgements}

We thank Solveig Grimstad and Susan Veldhof, Abbott Vascular, for advice and help with the infrastructure of the registry, and Divine Ediebah, Abbott Vascular, for statistical support.

\section{Funding}

This study was supported by an institutional grant from Abbott Vascular. A. Baumbach, A. Mathur and A. Lansky acknowledge the support of the Barts NIHR Biomedical Research Centre for this work.

\section{Conflict of interest statement}

A. Baumbach reports receiving an institutional research grant from Abbott Vascular. N. West is a consultant for and reports receiving research support from Abbott Vascular. A. Mathur has received an institutional research grant from Abbott Vascular. M. de Belder has received research support from Abbott Vascular. P. O'Kane has received speaker fees from Abbott Vascular. M. Egred has received speaker fees from Abbott Vascular. The other authors have no conflicts of interest to declare.

\section{References}

1. Onuma Y, Dudek D, Thuesen L, Webster M, Nieman K, Garcia-Garcia HM, Ormiston JA, Serruys PW. Five-year clinical and functional multislice computed tomography angiographic results after coronary implantation of the fully resorbable polymeric everolimus-eluting scaffold in patients with de novo coronary artery disease: the ABSORB cohort A trial. JACC Cardiovasc Interv. 2013;6:999-1009.

2. Cassese S, Byrne RA, Ndrepepa G, Kufner S, Wiebe J, Repp J, Schunkert H, Fusaro M, Kimura T, Kastrati A. Everolimuseluting bioresorbable vascular scaffolds versus everolimus-eluting metallic stents: a meta-analysis of randomised controlled trials. Lancet. 2016;387:537-44.

3. Collet C, Asano T, Miyazaki Y, Tenekecioglu E, Katagiri Y, Sotomi Y, Cavalcante R, de Winter RJ, Kimura T, Gao R, Puricel S, Cook S, Capodanno D, Onuma Y, Serruys PW. Late thrombotic events after bioresorbable scaffold implantation: a systematic review and meta-analysis of randomized clinical trials. Eur Heart J. 2017;38:2559-66.

4. Serruys PW, Chevalier B, Sotomi Y, Cequier A, Carrie D, Piek JJ, Van Boven AJ, Dominici M, Dudek D, McClean D, Helqvist S, Haude M, Reith S, de Sousa Almeida M, Campo G, Iniguez A, Sabaté M, Windecker S, Onuma Y. Comparison of an everolimus-eluting bioresorbable scaffold with an everolimus-eluting metallic stent for the treatment of coronary artery stenosis (ABSORB II): a 3 year, randomised, controlled, single-blind, multicentre clinical trial. Lancet. 2016;388:2479-91.

5. Ellis SG, Kereiakes DJ, Metzger DC, Caputo RP, Rizik DG, Teirstein PS, Litt MR, Kini A, Kabour A, Marx SO, Popma JJ, McGreevy R, Zhang Z, Simonton C, Stone GW; ABSORB III Investigators. Everolimus-Eluting Bioresorbable Scaffolds for Coronary Artery Disease. N Engl J Med. 2015;373:1905-15.

6. Rizik DG, Shah MG, Burke RF. First US experience following FDA approval of the ABBOTT vascular bioresorbable vascular scaffold for the treatment of coronary artery disease. Catheter Cardiovasc Interv. 2016;88:899-901.

7. Byrne RA, Serruys PW, Baumbach A, Escaned J, Fajadet J, James S, Joner M, Oktay S, Jüni P, Kastrati A, Sianos G, Stefanini GG, 
Wijns W, Windecker S. Report of a European Society of CardiologyEuropean Association of Percutaneous Cardiovascular Interventions task force on the evaluation of coronary stents in Europe: executive summary. Eur Heart J. 2015;36:2608-20.

8. Abizaid A, Ribamar Costa J Jr, Bartorelli AL, Whitbourn R, van Geuns RJ, Chevalier B, Patel T, Seth A, Stuteville M, Dorange C, Cheong WF, Sudhir K, Serruys PW; ABSORB EXTEND investigators. The ABSORB EXTEND study: preliminary report of the twelve-month clinical outcomes in the first 512 patients enrolled. EuroIntervention. 2015;10:1396-401.

9. Testa L, De Carlo M, Petrolini A, Rapetto C, Varbella F, Cortese B, Gabrielli G, Geraci S, Loi B, Boccuzzi G, Tarantini G, Fischetti D, Calabria P, Tomai F, Ribichini F, Tamburino C, Indolfi C, Bartorelli AL, Petronio AS, Bedogni F. One-year clinical results of the Italian diffuse/multivessel disease ABSORB prospective registry (IT-DISAPPEARS). EuroIntervention. 2017;13:424-31.

10. Nef H, Wiebe J, Achenbach S, Münzel T, Naber C, Richardt G, Mehilli J, Wöhrle J, Neumann T, Biermann J, Zahn R, Kastner J, Schmermund A, Pfannebecker T, Schneider S, Limbourg T, Hamm CW. Evaluation of the short- and long-term safety and therapy outcomes of the everolimus-eluting bioresorbable vascular scaffold system in patients with coronary artery stenosis: Rationale and design of the German-Austrian ABSORB RegIstRy (GABI-R). Cardiovasc Revasc Med. 2016;17:34-7.

11. Capodanno D, Gori T, Nef H, Latib A, Mehilli J, Lesiak M, Caramanno G, Naber C, Di Mario C, Colombo A, Capranzano P, Wiebe J, Araszkiewicz A, Geraci S, Pyxaras S, Mattesini A, Naganuma T, Münzel T, Tamburino C. Percutaneous coronary intervention with everolimus-eluting bioresorbable vascular scaffolds in routine clinical practice: early and midterm outcomes from the European multicentre GHOST-EU registry. EuroIntervention. 2015;10:1144-53.

12. Puricel S, Cuculi F, Weissner M, Schmermund A, Jamshidi P, Nyffenegger T, Binder H, Eggebrecht H, Munzel T, Cook S, Gori T. Bioresorbable Coronary Scaffold Thrombosis: Multicenter Comprehensive Analysis of Clinical Presentation, Mechanisms, and Predictors. J Am Coll Cardiol. 2016;67:921-31.

13. Cutlip DE, Windecker S, Mehran R, Boam A, Cohen DJ, van Es GA, Steg PG, Morel MA, Mauri L, Vranckx P, McFadden E, Lansky A, Hamon M, Krucoff MW, Serruys PW; Academic Research Consortium. Clinical end points in coronary stent trials: a case for standardized definitions. Circulation. 2007;115:2344-51.

14. Serruys PW, Onuma Y, Ormiston JA, de Bruyne B, Regar E, Dudek D, Thuesen L, Smits PC, Chevalier B, McClean D, Koolen J, Windecker S, Whitbourn R, Meredith I, Dorange C, Veldhof S, Miquel-Hebert K, Rapoza R, Garcia-Garcia HM. Evaluation of the second generation of a bioresorbable everolimus drug-eluting vascular scaffold for treatment of de novo coronary artery stenosis: six-month clinical and imaging outcomes. Circulation. 2010; 122:2301-12.

15. Geraci S, Kawamoto H, Caramanno G, Ruparelia N, Capodanno D, Brugaletta S, Gori T, Nef H, Sabate M, Mehilli J,
Lesiak M, Naber C, Di Mario C, Capranzano P, Wiebe J, Araszkiewicz A, Pyxaras S, Mattesini A, Munzel T, Tamburino C, Colombo A, Latib A. Bioresorbable Everolimus-Eluting Vascular Scaffold for Long Coronary Lesions: A Subanalysis of the International, Multicenter GHOST-EU Registry. JACC Cardiovasc Interv. 2017;10:560-8.

16. Ortega-Paz L, Capodanno D, Giacchi G, Gori T, Nef H, Latib A, Caramanno G, Di Mario C, Naber C, Lesiak M, Capranzano P, Wiebe J, Mehilli J, Araszkiewicz A, Pyxaras S, Mattesini A, Geraci S, Naganuma T, Colombo A, Munzel T, Sabaté M, Tamburino C, Brugaletta S. Impact of overlapping on 1-year clinical outcomes in patients undergoing everolimus-eluting bioresorbable scaffolds implantation in routine clinical practice: Insights from the European multicenter GHOST-EU registry. Catheter Cardiovasc Interv. 2017;89:812-8.

17. Wykrzykowska JJ, Kraak RP, Hofma SH, van der Schaaf RJ, Arkenbout EK, IJsselmuiden AJ, Elias J, van Dongen IM, Tijssen RYG, Koch KT, Baan J Jr, Vis MM, de Winter RJ, Piek JJ, Tijssen JGP, Henriques JPS; AIDA Investigators. Bioresorbable Scaffolds versus Metallic Stents in Routine PCI. $N$ Engl J Med. 2017;376:2319-28.

18. Ortega-Paz L, Capodanno D, Gori T, Nef H, Latib A, Caramanno G, Di Mario C, Naber C, Lesiak M, Capranzano P, Wiebe J, Mehilli J, Araszkiewicz A, Pyxaras S, Mattesini A, Geraci S, Naganuma T, Colombo A, Münzel T, Sabaté M, Tamburino C, Brugaletta S. Predilation, sizing and post-dilation scoring in patients undergoing everolimus-eluting bioresorbable scaffold implantation for prediction of cardiac adverse events: development and internal validation of the PSP score. EuroIntervention. 2017;12:2110-7.

19. Tanaka A, Latib A, Kawamoto H, Jabbour RJ, Sato K, Miyazaki T, Naganuma T, Mangieri A, Pagnesi M, Montalto C, Chieffo A, Carlino M, Montorfano M, Colombo A. Clinical outcomes of a real-world cohort following bioresorbable vascular scaffold implantation utilising an optimised implantation strategy. EuroIntervention. 2017;12:1730-7.

\section{Supplementary data}

Supplementary Table 1. Centres, principal investigators and enrolment numbers.

Supplementary Table 2. Predictors of 365-day TLF (protocol definition) results from univariable logistic regression - per subject analysis.

Supplementary Table 3. Predictors of 365-day MACE (protocol definition) results from univariable logistic regression - per subject analysis.

Supplementary Table 4. Predictors of 365-day ARC stent thrombosis results from univariable logistic regression - per subject analysis.

The supplementary data are published online at:

http://www.pcronline.com/

eurointervention/128th_issue/250 


\section{Supplementary data}

Supplementary Table 1. Centres, principal investigators and enrolment numbers.

\begin{tabular}{|c|c|c|}
\hline Papworth Hospital Cambridge & N. West & 150 \\
\hline Freeman Hospital Newcastle & A. Zaman & 119 \\
\hline Royal Bournemouth Hospital & P. O’Kane & 109 \\
\hline Bristol Heart Institute & T. Johnson & 91 \\
\hline Leeds General Infirmary & S. Wheatcroft & 67 \\
\hline James Cook Hospital Middlesbrough & M. de Belder & 58 \\
\hline Glan Clwyd & P. Das & 57 \\
\hline Northwick Park Hospital & A. El Ghamaz & 44 \\
\hline King’s College London & J. Byrne & 37 \\
\hline Queen Alexandra Hospital Portsmouth & H. Griffiths & 35 \\
\hline Barts Heart Centre London & M. Ozkor & 32 \\
\hline Norfolk \& Norwich University Hospital & T. Sarev & 30 \\
\hline Golden Jubilee Hospital Glasgow & M. Lindsay & 28 \\
\hline Glenfield Hospital Leicester & D. Adlam & 25 \\
\hline Royal Brompton University Hospital & R. de Silva & 24 \\
\hline Royal Sussex County Hospital Brighton & A. de Belder & 23 \\
\hline Royal Infirmary of Edinburgh & N. Uren & 19 \\
\hline Manchester Royal Infirmary & F. Fath-Ordoubadi & 17 \\
\hline John Radcliffe Hospital Oxford & A. Banning & 12 \\
\hline Royal Victoria Belfast & M. Spence & 11 \\
\hline Lister Hospital Stevenage & M. Srinivasan & 8 \\
\hline Basildon Hospital & A. Kabir & 7 \\
\hline Frimley Park Hospital & J. Shannon & 1 \\
\hline University Hospital Southampton & N. Curzen & 1 \\
\hline
\end{tabular}




\section{Supplementary Table 2. Predictors of 365-day TLF (protocol definition) results from univariable logistic regression - per subject analysis.}

\begin{tabular}{|c|c|c|c|c|}
\hline Variable & Coding for binary variables & Coefficient & $p$-value ${ }^{1}$ & Odds ratio $[95 \% \mathrm{CI}]$ \\
\hline Age (years) & & -0.04 & 0.0344 & $0.96[0.93,1.00]$ \\
\hline STEMI & Yes vs. no & -0.90 & 0.2219 & $0.41[0.10,1.72]$ \\
\hline Diabetes & Yes vs. no & 0.29 & 0.5033 & $1.34[0.57,3.15]$ \\
\hline Scaffold size & 2.5 vs. no 2.5 & 0.57 & 0.1174 & $1.78[0.87,3.65]$ \\
\hline Scaffold size & 3 vs. no 3 & -0.16 & 0.6481 & $0.85[0.42,1.72]$ \\
\hline Scaffold size & 3.5 vs. no 3.5 & 0.12 & 0.7375 & $1.13[0.56,2.30]$ \\
\hline Total scaffold length (mm) & & 0.01 & 0.1823 & $1.01[1.00,1.02]$ \\
\hline Medication & Clopidogrel vs. no clopidogrel & 0.56 & 0.1618 & $1.75[0.80,3.81]$ \\
\hline Medication & Ticagrelor or prasugrel vs. no ticagrelor or prasugrel & -0.55 & 0.1651 & $0.58[0.26,1.26]$ \\
\hline Bifurcation & Yes vs. no & -0.73 & 0.2330 & $0.48[0.15,1.60]$ \\
\hline Calcification & Moderate/severe vs. no & 0.70 & 0.1622 & $2.01[0.75,5.37]$ \\
\hline ACC/AHA lesion class & $\mathrm{B} 2$ or $\mathrm{C}$ vs. $\mathrm{A}$ or $\mathrm{B} 1$ & -0.38 & 0.2967 & $0.68[0.33,1.40]$ \\
\hline Number of stents implanted $>1$ per lesion & Yes vs. no & 0.46 & 0.2014 & $1.59[0.78,3.24]$ \\
\hline Post-dilatation & Yes vs. no & & CS & \\
\hline PSP & Yes vs. no & -0.01 & 0.9802 & $0.99[0.47,2.08]$ \\
\hline PSPR & Yes vs. no & 0.23 & 0.5281 & $1.26[0.62,2.56]$ \\
\hline DAPT at $1 \mathrm{yr}$ & Yes vs. no & 12.05 & 0.9929 & $171 \mathrm{E} 3[0.00,>999]$ \\
\hline Number of procedures per centre & $1-20$ vs. $>50$ & 0.26 & 0.6804 & $1.30[0.38,4.47]$ \\
\hline Number of procedures per centre & $21-50$ vs. $>50$ & 0.05 & 0.8945 & $1.06[0.47,2.35]$ \\
\hline Imaging & Yes vs. no & 0.53 & 0.1534 & $1.70[0.82,3.51]$ \\
\hline
\end{tabular}

1 by Wald chi-square test CS: complete separation 
Supplementary Table 3. Predictors of 365-day MACE (protocol definition) results from univariable logistic regression - per subject analysis.

\begin{tabular}{|c|c|c|c|c|}
\hline Variable & Coding for binary variables & Coefficient & $p$-value ${ }^{1}$ & Odds ratio $[95 \% \mathrm{CI}]$ \\
\hline Age (years) & & -0.03 & 0.0550 & $0.97[0.94,1.00]$ \\
\hline STEMI & Yes vs. no & -0.97 & 0.1885 & $0.38[0.09,1.61]$ \\
\hline Diabetes & Yes vs. no & 0.39 & 0.3446 & $1.48[0.66,3.32]$ \\
\hline Scaffold size & 2.5 vs. no 2.5 & 0.60 & 0.0919 & $1.82[0.91,3.66]$ \\
\hline Scaffold size & 3 vs. no 3 & -0.16 & 0.6533 & $0.85[0.43,1.70]$ \\
\hline Scaffold size & 3.5 vs. no 3.5 & 0.11 & 0.7625 & $1.11[0.56,2.21]$ \\
\hline Total scaffold length (mm) & & 0.01 & 0.1922 & $1.01[1.00,1.02]$ \\
\hline Medication & Clopidogrel vs. no clopidogrel & 0.64 & 0.1021 & $1.90[0.88,4.12]$ \\
\hline Medication & Ticagrelor or prasugrel vs. no ticagrelor or prasugrel & -0.64 & 0.1044 & $0.53[0.24,1.14]$ \\
\hline Bifurcation & Yes vs. no & -0.80 & 0.1903 & $0.45[0.14,1.49]$ \\
\hline Calcification & Moderate/severe vs. no & 0.86 & 0.0653 & $2.35[0.95,5.85]$ \\
\hline ACC/AHA lesion class & $\mathrm{B} 2$ or $\mathrm{C}$ vs. $\mathrm{A}$ or $\mathrm{B} 1$ & -0.36 & 0.3114 & $0.70[0.35,1.40]$ \\
\hline Number of stents implanted $>1$ per lesion & Yes vs. no & 0.48 & 0.1727 & $1.62[0.81,3.22]$ \\
\hline Post-dilatation & Yes vs. no & & CS & \\
\hline PSP & Yes vs. no & -0.05 & 0.8883 & $0.95[0.46,1.94]$ \\
\hline PSPR & Yes vs. no & 0.21 & 0.5435 & $1.24[0.62,2.46]$ \\
\hline DAPT at $1 \mathrm{yr}$ & Yes vs. no & 12.06 & 0.9926 & $172 \mathrm{E} 3[0.00,>999]$ \\
\hline Number of procedures per centre & $1-20$ vs. $>50$ & 0.16 & 0.7972 & $1.18[0.34,4.02]$ \\
\hline Number of procedures per centre & $21-50$ vs. $>50$ & -0.04 & 0.9121 & $0.96[0.43,2.10]$ \\
\hline Imaging & Yes vs. no & 0.50 & 0.1649 & $1.65[0.81,3.32]$ \\
\hline
\end{tabular}

${ }^{1}$ by Wald chi-square test.

CS: complete separation 


\section{Supplementary Table 4. Predictors of 365-day ARC stent thrombosis results from univariable logistic regression - per subject analysis.}

\begin{tabular}{|c|c|c|c|c|}
\hline Variable & Coding for binary variables & Coefficient & $p$-value ${ }^{1}$ & Odds ratio $[95 \% \mathrm{CI}]$ \\
\hline Age (years) & & -0.02 & 0.4705 & $0.98[0.94,1.03]$ \\
\hline STEMI & Yes vs. no & -1.02 & 0.3254 & $0.36[0.05,2.74]$ \\
\hline Diabetes & Yes vs. no & 0.88 & 0.0822 & $2.41[0.89,6.52]$ \\
\hline Scaffold size & 2.5 vs. no 2.5 & 1.18 & 0.0137 & $3.26[1.27,8.35]$ \\
\hline Scaffold size & 3 vs. no 3 & -0.03 & 0.9421 & $0.97[0.38,2.45]$ \\
\hline Scaffold size & 3.5 vs. no 3.5 & -0.14 & 0.7746 & $0.87[0.34,2.22]$ \\
\hline Total scaffold length (mm) & & 0.01 & 0.1369 & $1.01[1.00,1.03]$ \\
\hline Medication & Clopidogrel vs. no clopidogrel & 0.87 & 0.1277 & $2.38[0.78,7.30]$ \\
\hline Medication & Ticagrelor or prasugrel vs. no ticagrelor or prasugrel & -0.86 & 0.1296 & $0.42[0.14,1.29]$ \\
\hline Bifurcation & Yes vs. no & -1.29 & 0.2109 & $0.27[0.04,2.08]$ \\
\hline Calcification & Moderate/severe vs. no & 1.14 & 0.0497 & $3.11[1.00,9.67]$ \\
\hline ACC/AHA lesion class & $\mathrm{B} 2$ or $\mathrm{C}$ vs. $\mathrm{A}$ or $\mathrm{B} 1$ & -0.22 & 0.6502 & $0.80[0.32,2.06]$ \\
\hline Number of stents implanted $>1$ per lesion & Yes vs. no & 0.49 & 0.3112 & $1.62[0.64,4.16]$ \\
\hline Post-dilatation & Yes vs. no & & CS & \\
\hline PSP & Yes vs. no & -0.21 & 0.6706 & $0.81[0.31,2.12]$ \\
\hline PSPR & Yes vs. no & -0.26 & 0.5899 & $0.77[0.30,1.97]$ \\
\hline DAPT at $1 \mathrm{yr}$ & Yes vs. no & 11.03 & 0.9921 & $61635[0.00,>999]$ \\
\hline Number of procedures per centre & $1-20$ vs. $>50$ & 1.50 & 0.0166 & $4.47[1.31,15.2]$ \\
\hline Number of procedures per centre & $21-50$ vs. $>50$ & 0.57 & 0.2933 & $1.77[0.61,5.16]$ \\
\hline Imaging & Yes vs. no & 0.46 & 0.3433 & $1.59[0.61,4.13]$ \\
\hline
\end{tabular}

${ }^{1}$ by Wald chi-square test. 